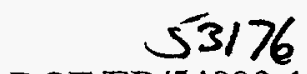

DOE/ER/S4093-12

\title{
Theoretical Plasma Physics
}

George Vahala and Eugene Tracy

RECEVED

\author{
Final Report \\ Finat Report \\ Department of Physics \\ College of William \& Mary \\ Williamsburg, VA 23187
}

JUL 191996

OSTI

April, 1996

DISCLAIMER

This report was prepared as an account of work sponsored by an agency of the United States Government. Neither the United States Government nor any agency thereof, nor any of their employees, makes any warranty, express or implied, or assumes any legal liability or responsibility for the accuracy, completeness, or usefulness of any information, apparatus, product, or process disclosed, or represents that its use would not infringe privately owned rights. Reference herein to any specific commercial product, process, or service by trade name, trademark, manufacturer, or otherwise does not necessarily constitute or imply its endorsement, recommendation, or favoring by the United States Government or any agency thereof. The views and opinions of authors expressed herein do not necessarily state or reflect those of the United States Government or any agency thereof.

PREPARED FOR THE U.S. DEPARTMENT OF ENERGY

UNDER GRANT NUMBER PE-FGQ2-STER 5993 DE-F605-84ERS 3176

Abstract

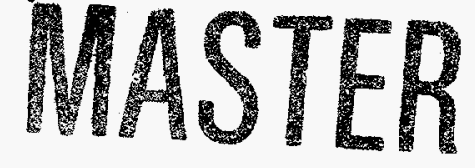




\section{DISCLAIMER}

Portions of this document may be illegible in electronic image products. Images are produced from the best available original document. 
During the past year, we have concentrated on (I) divertor physics, (II) thermo-lattice Boltzmann (TLBE) approach to turbulence, and (III) phase space techniques in gyro-resonance problems in collaboration with Dieter Sigmar (MIT), Sergei Krasheninnikov (MIT), Linda Vahala (ODU), Joseph Morrison (AS\&M/NASA-Langley), Pavol Pavlo and Josef Preinhaelter (Institute of Plasma Physics, Czech Academy of Sciences) and Allan Kaufman (LBL/U.C Berkeley).

Using a 2-equation compressible closure model with a 2D mean flow, we are investigating the effects of 3D neutral turbulence on reducing the heat load to the divertor plate by various toroidal cavity geometries. These studies are being extended to examine 3D mean flows

Thermal Lattice Boltzmann (TLBE) methods are being investigated to handle 3D turbulent flows in nontrivial geometries. It is planned to couple the TLBE collisional regime to the weakly collisional regime and so be able to tackle divertor physics.

In the application of phase space techniques to minority-ion RF heating, resonance heating is treated as a multi-stage process. A generalization of the Case-van Kampen analysis is presented for multi-dimensional non-uniform plasmas. Effects such as particle trapping and the ray propagation dynamics in tokamak geometry can now be handled using Weyl calculus.

\section{Table of Contents}

I. Effect of Neutral Fluid Turbulence in the Gas Blanket Region of the Divertor 2

II. TLBE Approach to Turbulence

III. Wave Propagation in Tokamaks $\quad 3$

IV. Chaotic Signal Analysis

V. Publications in 1995-1996 


\section{Effect of Neutral Fluid Turbulence in the Gas Blanket Region of the Divertor}

\section{[Ref. 1-3]}

Recent experiments and 2D laminar plasma-fluid simulations have indicated that plasma detachment from the divertor plate is strongly tied to plasma recombination. With plasma recombination, a neutral gas blanket will form between the divertor plate and the plasma flame front. Because of plasma-neutral coupling, the plasma flow along the field lines will drive neutral gas flow with Mach number $\geq 1$ and Reynolds number $\geq 1000$. A compressible set of conservation and transport equations are solved with 2D mean toroidal flow and 3D turbulence effects over various toroidal cavity geometries. The radial structure of the temperature profile is determined for both turbulent and laminar flow as the flame front propagates down the toroidal cavity. Quantitative results are obtained for the increased heat transfer to the toroidal walls due to turbulence as well as radial profiles for the transport coefficients. It is found that heat loads to the toroidal walls can be increased by factors of 5 to 20 over that for laminar flow over cavity geometries studied here. This increased heat transfer to the toroidal walls will lead to decreased levels of heat flux hitting the divertor plate.

\section{TLBE approach to turbulence [Ref. 4-5]}

Thermal Lattice Boltzmann (TLBE) techniques are used to consider the time evolution of free-decaying two dimensional (2D) turbulence induced by a double velocity shear layer. In particular, we consider the effect of this turbulence at a Reynolds number of 2555 on a strong temperature gradient. Since all structures are resolved on the 1024 x 1024 grid, the Smagorinsky model is employed to compute directly the eddy viscosity and eddy diffusivity. These transport coefficients play an integral part in large eddy simulations at very high Reynolds numbers where a direct simulation cannot resolve all excited scales. TLBE codes have the virtue of being readily extended to 3D, can readily handle non-periodic geometries, and are ideally suited for multiparallel computer architectures.

\section{Wave Propagation in Tokamaks [Ref. 6-11]}

It is found that the admixture of weak higher short-wavelength modes (several $\%$ of the coupled power) in the spatial power spectrum of the lower hybrid waves, radiated from the waveguide grill into an inhomogeneous scrape-off-layer plasma in tokamaks, has profound influence on the ponderomotive effects of these waves. Using barometric formula with the ponderomotive force potential for the electron density, we solve the coupling problem self consistently for 4 modes using shooting and matching method to fulfill surface impedance boundary conditions determined by infinite grill theory. For symmetric spectrum, below threshold, the total reflection coefficient is independent of the coupled power. Also, at these power levels, isolated density depressions (cavitons) are formed in the spatial locations where there is constructive superposition of short and long wavelength modes. Intense mutual mode conversion of these waves occur at these sites. For power levels above threshold the reflection coefficient increases till it saturates at higher powers. Numerical results are given for the 24 waveguide grill mounted on the ASDEX tokamak.

The emission of collective waves by a moving charged particle in a nonuniform medium is discussed. Emission occurs in a nonuniform medium when the em local dispersion relation of the collective wave is satisfied. This is a form of resonance crossing. Using the Weyl symbol calculus, a local expansion of the collective wave equation driven by the particle source is derived in the neighborhood of the crossing. The collective wave dispersion manifold and the gyroballistic wave dispersion manifold can be used as a pair of local coordinates in the neighborhood of the resonance crossing, which greatly simplifies the analysis. This change of representation is carried out using a metaplectic transform (a generalization of the fourier transform). The Wigner function of the emitted wave field is then computed in the new coordinates. The Wigner function is a phase space scalar, hence the numerical value is invariant under linear canonical transformations. This invariance is invoked to finally arrive at the Wigner 
function in the original (physical) coordinates. The wave-action and -energy emission rates are then computed from the Wigner function.

An inverted population of energetic minority ions, such as neonatal alphas, can support a Bernstein wave at any harmonic of their gyrofreqency. The sign of the wave energy depends on the local wave-vector, whose propagation rate is proportional to the magnetic-field gradient. Two important linear-conversion phenomena result from this propagation: (1) When a Bernstein ray crosses the gyroresonance layer, its energy flips sign. We show that this results in energy transfer to gyroballistic rays, with a conversion coefficient exactly equal to two. (2) When a negativeenergy Bernstein ray crosses the magnetosonic dispersion surface, its energy becomes exponentially increased by a modest factor, and simultaneously a magnetosonic ray is produced. We derive the linear convective saturation of the resulting magnetosonic-cyclotron instability, previously studied for a uniform magnetic field. We also obtain this saturation for a oblique magnetosonic wave, due to gyroresonance with the energetic minority.

The generalization of the Case-van Kampen analysis to multi-dimensional non-uniform plasma is presented. Application of this analysis is made to minority-ion gyroresonant heating in an axisymmetric tokamak. In previous work, the Case-van Kampen analysis, in conjunction with the Bateman-Kruskal algorithm, was used in a one-dimensional slab model to compute the collective wave spin-off (to the minority-ion Bernstein wave) and the gyroballistic continuum for minority gyroresonant absorption. The generalization to multidimensions and non-trivial geometries requires several important new developments: In tokamak geometry particles can be trapped, an effect which is absent in the slab model. The ray propagation dynamics for both the free gyroballistic waves and the collective minority-ion Bernstein wave is far more complicated than in the slab model. In particular, a resonance zone is identified wherein the gyroballistic waves interact strongly and cannot be treated as free. We use the Weyl calculus to construct a local form of the self-consistent gyroballistic equation within the resonance zone. This reduced equation is simplified via a metaplectic transformation (a generalization of the fourier transformation). After this simplification, the equation is shown to be of Case-van Kampen type with weak non-uniformities, hence there are no true Case-van Kampen eigenfuctions. Using the Bateman-Kruskal approach, a local Case-van Kampen basis can be constructed and the initial value problem solved. The self-consistent interactions of the gyroballistic continuum lead to a collective wave, the minority-ion Bernstein wave. The Bernstein wave is extracted by the spectral deformation approach of Crawford and Hislop. The relevance of this work to the theory of collective phenomena in nonlinear oscillator ensembles is briefly discussed.

\section{Chaotic Signal Analysis [Ref. 12]}

A method is discussed for reconstructing chaotic systems from noisy signals using a symbolic approach. The state space of the system is partitioned into subregions and a symbol is assigned to each subregion. Consequently, an oribt in a continuous space is converted into a long symbol string. The probabilities of occurrence for different symbol sequences constitute the symbol sequence statistics. The symbol sequence statistics are easily measured from the signal output and are used as the target for reconstruction (i.e. for assessing the goodness of fit of proposed models). Reliable reconstructions were achieved given a noisy chaotic signal, provided the general class of the model of the underlying dynamics is known. Both observational and dynamical noise were considered, and they were not limited to small amplitudes. Substantial noise produces a strong bias in the symbol sequence statistics, but such bias can be tracked and effectively eliminated by including the noise characteristics in the model. This is demonstrated by the robust reconstruction of the Henon and Ikeda maps even when the signal to noise ratio is approximately 1. Applications of this method include extracting control parameters for nonlinear dynamical systems and nonlinear model evaluation from experimental data. 


\section{Publications in $\mathbf{1 9 9 5 - 9 6}$}

1. "Effects of Neutral 3D Turbulence in the Gas Blanket Regime for Divertors" G. Vahala, L. Vahala, J. Morrison, S. Krasheninnikov and D. Sigmar Phys. Lett A205, 266 (1995)

2. "Toroidal Wall Heat Flux and Conductivity Profiles due to Heutral 3D Turbulence in the Gas Blanket Regime for Divertors"

G. Vahala, L. Vahala, J. Morrison, S. Krasheninnikov and D. Sigmar Contr. Plasma Physics (to be published)

3. "K- $\mathrm{K}$ Compressible 3D Neutral Fluid Turbuelnce Modeling of the Effect of Toroidal Cavities on Flame Front Propagation in the Gas Blanket Regime for Tokamak Divertors"

G. Vahala, L. Vahala, J. Morrison, S. Krasheninnikov \& D. Sigmar

J. Plasma Phys. (submitted)

4. "Effect of Velocity Shear on a Strong Temperature Gradient - a Lattice Boltzmann Approach" G. Vahala, P. Pavlo, L. Vahala \& H. Chen

Phys. Lett. A202, 376 (1995)

5. "Determineation of Eddy Transport Coefficients in Thermo-Lattice Boltzmann Modeling of

Two-Dimensional Turbulence"

G. Vahala, P. Pavlo, L. Vahala and M. Soe

Czech J. Phys. (to be published)

6. "Large Quasi-Optical Grills Mounted in Hyperguides"

J. Preinhaelter, L. Vahala \& G. Vahala

Proc. 17th Symp. on Plasma Physics and Technology (Prague 1995, June 13-16), p. 43

7. "Slow Lower Hybrid Wave Pondermotive Effects in the Edge of a Tokamak Plasma"

J. Preinhaelter, L. Vahala, and G. Vahala

in 1lth Topical Conference on Radio Frequency Power in Plasmas, ed. Ronald

Prater \& Vincent S. Chan, A.I.P Conf. Proc.No. 355 (1995), p. 255

8. "Role of the Higher Spatial Harmonics in the Launched LH Spectrum in the Ponderomotive Effects at the Plasma Edge"

J. Preinhaelter, L. Vahala \& G. Vahala

J. Plasma Phys. (to be published); IPPCZ-Report 350 (Dec. 1995)

9. "Wave emission by resonance crossing"

E.R. Tracy, A.N. Kaufman and Y. -M. Liang

Phys. Plasmas 2, 4413 (1995)

10. "Negative-energy energetic-ion Bernstein-wave propagation in a nonuniform magnetic field: two linear-conversion phenomena"

A. N. Kaufman, A. J. Brizard and E. R. Tracy

in the proceedings of the 11th Topical Conference on RF Power in Plasmas, March 1995.

11. "Generalized Case-van Kampen modes in multi-dimensional non-uniform plasma with application to gyroresonance heating"

E.R. Tracy, A.J. Brizard and A.N. Kaufman

J. Plasma Phys. (to be published) 
12. "Symbol sequence statistics in noisy chaotic signal reconstruction" X. Z. Tang, E. R. Tracy, A. D. Boozer, A. deBrauw and R. Brown Phys. Rev. E51, 3871 (1995). 